\title{
Les objets « van Grecken ». De bien ténébreuses affaires. Présentation
}

\section{Philippe Peltier}

\section{(2) OpenEdition \\ Journals}

Édition électronique

URL : http://journals.openedition.org/jso/7527

DOI : $10.4000 /$ jso. 7527

ISSN : 1760-7256

Éditeur

Société des océanistes

Édition imprimée

Date de publication : 31 décembre 2016

Pagination : 176-178

ISSN : 0300-953x

\section{Référence électronique}

Philippe Peltier, « Les objets « van Grecken ». De bien ténébreuses affaires. Présentation », Journal de la Société des Océanistes [En ligne], 142-143 | 2016, mis en ligne le 31 décembre 2016, consulté le 24 septembre 2020. URL : http://journals.openedition.org/jso/7527 ; DOI : https://doi.org/10.4000/jso 7527 


\section{Les objets "van Grecken ». De bien ténébreuses affaires}

\section{Philippe PELTIER, Harry BERAN et Nicolas GARNIER}




\title{
Présentation
}

\author{
par
}

\author{
Philippe PELTIER*
}

Lors de la création du musée des Arts africains et océaniens au début des années 1960, le professeur Jean Guiart, alors responsable de la section Océanie, effectua plusieurs missions d'acquisition dans le Pacifique. En 1965, il se rendit dans la vallée du Sepik, en Papouasie NouvelleGuinée, et en Australie où il visita marchands et collectionneurs. Il effectua une autre mission d'achat quelques dix ans plus tard. L'objet de ces missions était de constituer un fonds de collection sur une région qui n'était pas représentée dans l'ancien musée de la France d'Outre-mer dont le tout jeune musée était l'héritier. Lors de ces voyages, le professeur Guiart rendit visite par deux fois à Gene van Grecken, un marchand de Sydney. Il lui acheta un ensemble de pièces étonnantes dont une première série de quatorze sculptures qui firent rapidement l'objet d'une polémique quant à leur authenticité. L'article d'Harry Beran que l'on lira à la suite revient sur cette histoire mais surtout démontre pourquoi, à ses yeux, cette collection est un canular réalisé afin de tromper les experts. La rédaction de cette étude remonte à dix ans. Pour diverses raisons, elle ne fut jamais publiée. Harry Beran la fit lire aux deux protagonistes. La réaction fut violente. On s'attendait difficilement à moins !

Il nous parait cependant utile de revenir brièvement sur les débats qui entourent la collection que nous désignerons ici sous le nom de son inventeur (au sens de découvreur!) " van Grecken ", car elle occupe, dans les écrits du professeur Guiart sur l'art, une place particulière.
Alors que ce dernier, auteur prolifique, ne publiera jamais aucune réflexion sur sa mission dans le Sepik et sur ses choix d'objets, il reviendra par deux fois - comme le signale Beran dans son introduction sur les "van Grecken ». La première étude parait en 1967 dans un numéro du Journal de la Société des Océanistes, la seconde en 1969 dans les actes d'un colloque qui eut lieu à Los Angele et qui réunissait toutes les têtes pensantes sur l'art primitif de l'époque. Remarquons que ce second article est beaucoup plus linéaire et descriptif que le premier.

L'insistance du professeur Guiart à publier les "van Grecken » semble avoir pour but principal d'affirmer que ces objets ne sont pas des faux comme le bruit court déjà à l'époque, mais une découverte fondamentale qui remettait en cause au moins la connaissance des arts du Sepik, si ce n'est l'approche théorique des arts primitifs. Les "van Grecken ", objets dont des équivalents n’ont jamais été observés ou collectés, seraient les indices d'une tradition stylistique parallèle à celles connues et répertoriées jusqu'au début des années 1960. Leurs lieux de création - difficiles à déterminer mais que le professeur Guiart suppose de régions différentes et même parfois éloignées -, tout comme leurs formes sont la preuve que les zones stylistiques ne sont jamais fixes et que toute tentative pour les déterminer est vouée à l'échec ${ }^{1}$. En arrière-plan cette découverte remettait en cause les collectes effectuées par Alfred Bühler en 1956 et en 1959, mais aussi la carte stylistique qu'il venait de publier (Bühler, 1960). Pour le professeur Guiart une telle carte avec ses frontières

1. Jean Guiart reviendra sur le sujet dans une longue lettre qu'il adressa à D. Moore, conservateur à l'Australian Museum de Sydney. Les mêmes arguments sont repris dans un texte dactylographie intitulé « De l'interprétation stylistique en matière d'art primitif ", archives Roger Bastide, Musée du quai Branly, numéro D003591/46252.

* Conservateur général du Patrimoine, UP Océanie-Insulinde, musée du quai Branly, ppe@quaibranly.fr 
ne pouvait rendre compte des très nombreux échanges entre les groupes et de la circulation intense des objets ainsi que des très nombreux changements intervenus dans la production depuis la colonisation allemande. Bühler ne disait d'ailleurs pas autre chose dans son texte mais la carte traduisait mal cette fluidité par des lignes pointillées. Mais pour le profeseur Guiart le point important était que Bühler n'avait pas compris qu'il existait une forme d'expression qui ignorait les frontières.

Pour le professeur Guiart, il fallait donc reprendre les études à zéro et travailler non plus par zones mais village par village (une idée d'ailleurs reprise de Reimar Schefold dont les travaux furent publiés en 1966). Travail titanesque que personne n'a pu porter à son terme.

Est-ce aller trop loin que d'imaginer que la collection van Grecken était une trop belle occasion de s'inscrire dans la lignée des découvreurs de formes jusqu'alors inconnues? On peut imaginer le frisson délicieux ressenti par un chercheur, voulant laisser son nom dans l'histoire lors de la découverte d'une collection de pièces inconnues (cette découverte était d'ailleurs le rêve de tout marchand d'art primitif dans ces années où le monde se vidait de ses objets). Une collection qui se révélait correspondre avec les théories du professeur et se présentait comme une trop belle opportunité: c'était la parfaite rampe de lancement qui permettait à la section Océanie dont il était conservateur, du tout jeune musée parisien de devenir un centre de recherche indispensable et de reprendre la main sur les études et de damer le pion à Bâle, centre d'étude alors le plus dynamique!

Il faut cependant remettre cette découverte en perspective. La connaissance sur le Sepik, dans ces années, n'était pas ce qu'elle est de nos jours. Il existait alors peu de publications sur le sujet. Les grandes références étaient les livres de Schlaginhaufen (1910a-b) et de Reche (1913), deux expéditions d'avant la Première Guerre mondiale organisées respectivement pas le musée d'ethnographie de Dresde et la seconde par celui de Hambourg. Quant aux collections de Berlin, constituées à la même période mais restées invisibles depuis la fin de la Seconde Guerre mondiale, leur édition par Heinz Kelm était en cours (Kelm, 1966a-b, 1968, 1969). Enfin, comme le montrait l'exposition à la d'Arcy Gallery de New York sur l'art du Korewori en 1968, des découvertes étaient toujours possibles (Haberland, 1968)2 .
L'origine géographique des objets, le fait que cette origine débordait la simple vallée du Sepik, aurait dû alerter le professeur Guiart. Plusieurs personnes l'en avertirent dont Moore, conservateur à l'Australian Museum de Sydney, qui dans une lettre de novembre 1968, l'avertissait que les pièces étaient d'origine douteuse ${ }^{3}$. Guiart trouva à ceci une explication qu'il développe dans l'article paru dans le Journal de la Société des Océanistes (1967: 8 sqq). Il s'appuie sur une analyse du texte d'Anthony Forge sur le style. Guiart affirme qu'il existe bien en NouvelleGuinée un art fait d'imbrication de figures ou de recomposition de corps - ce que l'on ne peut remettre en doute. Et dans cette recomposition l'élément sexuel - ou pour reprendre son expression " une sensualité équivoque " - joue un rôle fondamental. Que cette sensualité équivoque s'exprime sur des bouchons de flûtes (c'est ainsi que le professeur Guiart classe ces objets), rien de plus normal à ses yeux puisque ces items sont tout à la fois l'expression de la virilité et de la communion masculine, communion qui trouve aussi son expression dans le mélange sur le sol de la salive dû à la mastication de la noix verte de bétel... Il fallait y penser! Cette assimilation entre objet, virilité et crachat et d'autant plus étonnant que, dans le Sepik, les hommes, occupant chacun une place spécifique, font très attention de laisser couler la salive produite par la mastication du bétel entre les lattes disjointes du plancher qui est surélevé par rapport au sol.

Dans le long temps qui sépare la rédaction et la publication de son article, Harry Beran a eu une longue correspondance avec van Grecken et le professeur Guiart. Celui-ci donna finalement son accord à sa publication ${ }^{4}$. Il demanda aussi que soient effectuées des analyses scientifiques. Ce qui fut finalement fait ${ }^{5}$. Les méthodes d'analyse employées ne suivent cependant pas les recommandations faites par le professeur Guiart, recommandations dont la naïveté laisse a sourire toute personne un peu versée dans le domaine. On lira ci-après un résumé des résultats obtenus à la suite des analyses très fines menées sur les pigments par Nicolas Ganier et sur les bois par Victoria Asensi Amoros. Ils confirment, non pas sur un fait mais sur un faisceau de faits que nous sommes bien en face de faux. Citons quelques exemples. Certains bois ne se retrouvent pas en NouvelleGuinée comme Shorea, une espèce du sud-est asiatique. C'est le bois utilisé pour "L'homme picoré par les oiseaux» (72.1966.14.1). Ou

2. Notons cependant quelques pièces de cette région étaient connues. La plus ancienne est celle conservée au musée de Brême sous le numéro D 3905. Le professeur Guiart en acheta aussi lors de sa mission.

3. Lettre conservée aux archives du musée du quai Branly : D001591/46136.

4. «The Beran paper should be published, for the record and left at that for the present. " note 5 in Fakes of Oceania, A Retort. Article dactylographie envoyé au Président du musée du quai Branly, non daté.

5. Rapport d'expertise xylologique, mars 2014 par le Dr Victoria Asensi Amoraos, UPMC-Paris VI, 16p. Nicolas Garnier, Analyse de la polychromie d'une série de Statuettes du Sepik. SAS Laboratoire Nicolas Garnier, décembre 2013, 81 p. Dactylographies. Ces deux rapports sont consultables au musée du quai Branly. 
encore Peronema canescens, un bois d'Insulinde utilisé pour la figure féminine 72.1976.1.8. Autre exemple : l'utilisation d'un produit lacté - plus certainement du beurre!-comme liant pour les pigments. À ma connaissance ni le beurre ni le lait n'existaient dans le Sepik (sauf introduit par les colons, mais il faut se rappeler que ces pièces seraient anciennes et que lors de leur supposée collecte, seule une quinzaine de colons vivaient dans le Sepik !). De même la présence d'huile de muscade sur plusieurs objets (dont 72.1966.14.1), est pour le moins curieuse. Cette huile, à ma connaissance, ne circula jamais dans la région. Ou encore de la peinture antirouille. Si l'on peut avancer que les artistes locaux ont pu à un moment se procurer de la peinture antirouille (ferrite de zinc) auprès de colons, cette peinture indique les objets n’ont pas été fabriqués avant 1930 et qu'ils ne peuvent donc pas provenir des collections auxquelles le vendeur les attribue. Ou encore l'usage d'une cire saponifiée qui ne se trouve pas à l'état naturel et qui fut lessivée avec un produit alcalin (par exemple sur 72.1968.14.10). Ou d'un noir de carbone utilisant une graisse animale comme liant, ce qui est un matériau classique chez les restaurateurs d'œuvre d'art. La présence de ces matériaux sur certaines couches indique toute une cuisine qui était propre aux restaurateurs de l'ancienne génération et aux faussaires qui voulaient donner une «belle et trompeuse patine aux objets". Certains pigments sont d'origine chimique. D'autres sont rares. Là encore à moins d'imaginer qu'un marchand de couleurs pour artiste se soit installé dans le Sepik... Enfin il y a plus troublant. Deux objets présentent exactement les mêmes constituants dans leur patine. Il s'agit de 72.1966,14.13 et de 72.1966,14.10. Or l'un est attribué à la région Asmat, l'autre à la vallée du Sepik. Ces données sont suffisamment évidentes pour affirmer que la collection est l'œuvre d'un faussaire, quelque fut ce faussaire.

Cette belle collection defaux fait partiemaintenant de l'histoire. Elle pose un cas intéressant d'objets dont le pouvoir de fascination et de séduction de par leur charge ambiguë a parfaitement joué son rôle. Elle marque un moment des études sur l'art du Sepik mais aussi de l'histoire du goût. Elle est d'accord avec l'idée que le Sepik est un lieu étonnant de création - ce qu'il est effectivement où tout est toujours possible. Ce qui est aussi vrai. Et c'est justement parce que tout était possible, que de tels objets furent considérés par le professeur Guiart comme des objets authentiques. Mais le possible a toujours des limites.

Août 2016

\section{BIBLIOGRAPHIE}

BuHLer Alfred, I960. Kunststile am Sepik: Führer durch das Museum für Völkerkunde und Schweiz. Museum für Volkskunde Basel Sonderausstellung vom 11 Juni bis 30 November.

Guiart Jean, 1967. Art primitifs et «structures ", Journal de la Société des Océanistes 23, pp. 1-17.

- 1969. The Concept of Norm in the Art of Some Oceanian Societies, in Daniel Biebuyck (ed.), Tradition and Creativity in Tribal Art, Universtity of Califormania Press, pp. 84-97.

Haberland Eike. 1968. The Caves of Karawari Mountains, New York, D’Arcy Galleries.

Kelm Heinz. 1966a. Kunst vom Sepik I [Mittellauf], Museum für Völkerkunde Berlin, Veröffentlichungen des Museums für Völkerkunde N. F. 10.

—, 1966b. Kunst vom Sepik II [Oberlauf], Museum für Völkerkunde Berlin, Veröffentlichungen des Museums für Völkerkunde N. F. 11.

—, 1968. Kunst vom Sepik III [Unterlauf \& Nachträge], Museum für Völkerkunde Berlin, Veröffentlichungen des Museums für Völkerkunde N. F. 15.

—, 1969. Kunstgegenstände aus dem Sepik-Gebiet (Neuguinea), Baessler-Archiv 17 (2), pp. 303364.

Reche Otto. 1913. Der Kaiserin-Augusta-Fluss, Hamburg, L. Friederichsen \& Co.

SCHEFold Reimar, 1966. Versuch einer Stilanalyse der Aufhängehaken vom mittleren Sepik in Neu-Guinea, hrsg. von der Geographisch-Ethnologischen Gesellschaft, Basel, Basel und dem Museum für Völkerkunde und Scweizerischen Museum für Volkskunde.

Schlaginhaufen Otto. 1910a. Reisen in Kaiser-Wilhelmsland (Neuguinea), in Abhandlungen und Berichte des Königl. Zoologischen und Anthropologisch-Ethnographischen Museums zu Dresden 13 (1), pp. 1-19.

—, 1910b. Eine ethnographische Sammlung vom Kaiserin- Augustafluss in Neuguinea, Abhandlungen und Berichte des Königl. Zoologischen und Anthropologisch-Ethnographischen Museums zu Dresden 13 (2), pp. II-III et pp. 1-74. 